\title{
Estilo de dirección y calidad de la gestión en la Institución Educativa Domingo Mandamiento Sipan - Hualmay- 2017
}

\section{Style Of Management And Quality Of Management in the Institución Educativa Domingo Mandamiento Sipan - Hualmay- 2017}

\begin{abstract}
Armando Graus Mesta
\section{RESUMEN}

Objetivo. Conocer la relación del estilo de dirección y la calidad de la gestión educativa en la Institución Educativa Domingo Mandamiento Sipán Hualmay - 2017. Material y Método. Investigación de enfoque cuantitativo, de tipo básica, nivel relacional, diseño no experimental correlacional de corte transversal, la población y muestra estuvo conformada por 60 docentes de la Institución Educativa. Resultados. El $53 \%$ de los docentes percibieron una gestión educativa alta, así mismo el $60 \%$ sintieron un estilo de dirección alto, por otro lado al estudiar las dimensiones de los estilos de dirección se pudo apreciar que el $62 \%$ de los docentes percibieron un estilo autocrático, el $57 \%$ estilo democrático y el $63 \%$ estilo liberal Conclusiones. Al contrastar la hipótesis de la investigación con la prueba no paramétrica Rho de Spearman se llegó a comprobar que existe relación directa y significativa entre el estilo de dirección y la calidad de la gestión educativa en la Institución Educativa Domingo Mandamiento Sipán - Hualmay-2017.
\end{abstract}

Palabra Clave: Estilo de dirección y calidad de la gestión.

\begin{abstract}
Objective. Know the relationship between the management style and the quality of educational management in the Educational Institution Domingo Mandamiento Sipan - Hualmay - 2017. Materials andMethod. Research ofi quantitative approach, basic type, relational level, non-experimental crosssectional correlational design, the population and sample consisted ofi60 teachers ofithe Educational Institution. Results $53 \%$ of teachers perceived a high educational management, likewise $60 \%$ felt a high management style, on the other hand when studying the dimensions of management styles it could be seen that $62 \%$ of teachers perceived an autocratic style, $57 \%$ democratic style and $63 \%$ liberal style Conclusions. When contrasting the research hypothesis with the non-parametric Spearman Rho test, it was found that there is a direct and significant relationship between the management style and the quality of educational management in the Educational Institution Domingo Mandamiento Sipan - Hualmay-2017
\end{abstract}

Key words: Management style and management quality

\section{INTRODUCCIÓN}

En la actualidad se sabe que el estilo de dirección alude a la manera específica por la cual un individuo responsable de un procedimiento autorizado desarrolla su trabajo. Es claro, por lo tanto, que diversos estilos de dirección pueden influir en la gestión, con el argumento de que hay algunos estilos que mejor se adaptan a los objetivos generales del procedimiento.

Desde los primeros estudios sobre eficacia escolar, la Dirección del Centro ha sido uno de los factores escolares mayormente señalados. Con distintas acepciones y matizaciones, las investigaciones sobre eficacia escolar han ido caracterizando la función de la dirección principalmente en torno al liderazgo. Acepciones tales como liderazgo institucional, educativo, profesional, firme y dirigido, estable, de apoyo, etc., representan constructos frecuentemente recurrentes para denotar al factor más indicativo de la dirección asociado a la eficacia escolar

Gómez (2013) en su investigación Incidencia del estilo de gestión escolar en el clima institucional, un estudio de casos, Universidad Abierta Interamericana llegando a la conclusión que el estilo de gestión escolar incide con el clima institucional.
Arana (2017) en su investigación Liderazgo directivo y desempeño docente en una Institución Educativa Parroquial del Distrito de San Isidro Lima - Perú, llegando a la conclusión que existe una relación directa y significativa entre estas dos variables, señalando que el estilo que más predomina es el Liderazgo Transformacional y un alto nivel de desempeño de los docentes en la institución educativa.

La presente investigación se justifica por la necesidad que se tiene de conocer los factores que influyen en la crisis de la calidad de la gestión de la institución educativa estatal. Esta situación de crisis no es sólo de tipo administrativo, sino que en lo profundo es de tipo pedagógico. Sin embargo, reconocemos que el sistema de soporte constituye un elemento básico que permitirá sentar las bases para superar esta situación deficitaria.

Por tal motivo, el objetivo de este estudio fue conocer la relación del estilo de dirección y la calidad de la gestión educativa en la Institución Educativa Domingo Mandamiento Sipán-Hualmay-2017.

\section{MATERIALES Y MÉTODOS}

La metodología aplicada en la presente investigación fue de tipo básica, el nivel fue correlacional, el diseño no experimental de

Recibido:09/10/2020 - Aprobado:19/11/2020

Universidad Nacional José Fustino Sanchez Carrión (Huaura - Perú). 
carácter transversal.

La población estuvo conformada por 60 docentes de la Institución Educativa Domingo Mandamiento Sipán Hualmay, por consiguiente como muestra se consideró todo el universo de la población.

El instrumento que se empleó fue el cuestionario, que según Ávila (2010) indica que "es un instrumento constituido por un conjunto de preguntas sistemáticamente elaboradas, que se formulan al encuestado o entrevistado, con el propósito de obtener los datos de las variables consideradas en el estudio" (p.38).

El cuestionario aplicado presentaba diecisiete (17) preguntas cerradas, las cuales permitieron ecoger la información sobre el Estilo de dirección y Calidad de la Gestión de los docentes de la Institución Educativa Domingo Mandamiento Sipán - Hualmay, todas las respuesta tienen un grado de intensidad que representa una escala de uno (1) a cinco (5), teniendo como criterio siguiente: Siempre (5), Casi siempre (4), A veces (3), Casi nunca (2) y Nunca (1).

Con respecto a técnica estadística, se utilizó el coeficiente de correlación Rho de Spearman a su vez fueron procesados en el programa estadístico SPSS V22 versión prueba.

\section{RESULTADOS}

En la tabla 1 se observa que el $43 \%$ de los docentes encuestados percibieron un alto estilo de dirección así mismo una alta gestión educativa.

Tabla 1.

Estilo de dirección según calidad de la gestión educativa.

\begin{tabular}{|c|c|c|c|c|c|c|c|c|}
\hline \multirow{3}{*}{$\begin{array}{l}\text { Estilo de } \\
\text { dirección }\end{array}$} & \multicolumn{6}{|c|}{ Gestión esducativa } & \multicolumn{2}{|c|}{ TOTAL } \\
\hline & \multicolumn{2}{|c|}{ Bajo } & \multicolumn{2}{|c|}{ Medio } & \multicolumn{2}{|c|}{ Alto } & & \\
\hline & $\mathrm{N}^{\circ}$ & $\%$ & $\mathrm{~N}^{\circ}$ & $\%$ & $\mathrm{~N}^{\circ}$ & $\%$ & $\mathrm{~N}^{\circ}$ & $\%$ \\
\hline Bajo & 1 & 2 & 5 & 8 & 2 & 8 & 8 & 13 \\
\hline Medio & 2 & 3 & 10 & 17 & 4 & 16 & 16 & 27 \\
\hline Alto & 3 & 5 & 7 & 12 & 26 & 36 & 36 & 60 \\
\hline TOTAL & 6 & 8 & 22 & 37 & 32 & 60 & 60 & 100 \\
\hline
\end{tabular}

En la tabla 2 se observa que el $45 \%$ de los docentes encuestados percibieron un alto estilo de dirección autocrático, así mismo fue la calidad de gestión educativa.

\section{Tabla 2}

Estilo autocrático y calidad de gestión educativa

\begin{tabular}{llllllllll}
\hline \multirow{2}{*}{$\begin{array}{l}\text { Estilo de } \\
\text { dirección }\end{array}$} & \multicolumn{3}{l}{ Gestión esducativa } & \multicolumn{3}{c}{ TOTAL } \\
\cline { 2 - 7 } & \multicolumn{3}{c}{ Bajo } & \multicolumn{3}{c}{ Medio } & \multicolumn{3}{c}{ Alto } \\
& \multicolumn{1}{l}{$\mathrm{N}^{\circ}$} & $\%$ & $\mathrm{~N}^{\circ}$ & $\%$ & $\mathrm{~N}^{\circ}$ & $\%$ & $\mathrm{~N}^{\circ}$ & $\%$ \\
\hline Bajo & 1 & 2 & 3 & 5 & 2 & 3 & 8 & 10 \\
Medio & 3 & 5 & 11 & 18 & 3 & 5 & 16 & 28 \\
Alto & 2 & 3 & 8 & 14 & 27 & 45 & 36 & 62 \\
TOTAL & 6 & 8 & 22 & 37 & 32 & 53 & 60 & 100
\end{tabular}

En la tabla 3 se observa que el $42 \%$ de los docentes encuestados percibieron alto estilo de dirección democrática, así mismo una alta calidad de la gestión educativa.

Tabla 3

Estilo democrático y calidad de gestión educativa

\begin{tabular}{|c|c|c|c|c|c|c|c|c|}
\hline \multirow{3}{*}{$\begin{array}{l}\text { Estilo de } \\
\text { dirección }\end{array}$} & \multicolumn{6}{|c|}{ Gestión esducativa } & \multicolumn{2}{|c|}{ TOTAL } \\
\hline & \multicolumn{2}{|c|}{ Bajo } & \multicolumn{2}{|c|}{ Medio } & \multicolumn{2}{|c|}{ Alto } & & \\
\hline & $\mathrm{N}^{\circ}$ & $\%$ & $\mathrm{~N}^{\circ}$ & $\%$ & $\mathrm{~N}^{\circ}$ & $\%$ & $\mathrm{~N}^{\circ}$ & $\%$ \\
\hline Bajo & 10 & 0 & 2 & 3 & 3 & 5 & 5 & 8 \\
\hline Medio & 4 & 7 & 13 & 22 & 4 & 6 & 21 & 35 \\
\hline Alto & 2 & 3 & 7 & 12 & 25 & 42 & 34 & 57 \\
\hline TOTAL & 6 & 10 & 22 & 37 & 32 & 53 & 60 & 100 \\
\hline
\end{tabular}

En la tabla 4 se observa que el $46 \%$ de los docentes encuestados percibieron alto estilo de dirección liberal, así mismo una alta calidad de la gestión educativa.

Tabla 4

Estilo liberal y calidad de gestión educativa

\begin{tabular}{|c|c|c|c|c|c|c|c|c|}
\hline \multirow{3}{*}{$\begin{array}{l}\text { Estilo de } \\
\text { dirección }\end{array}$} & \multicolumn{6}{|c|}{ Gestión esducativa } & \multicolumn{2}{|c|}{ TOTAL } \\
\hline & \multicolumn{2}{|c|}{ Bajo } & \multicolumn{2}{|c|}{ Medio } & \multicolumn{2}{|c|}{ Alto } & & \\
\hline & $\mathrm{N}^{\circ}$ & $\%$ & $\mathrm{~N}^{\circ}$ & $\%$ & $\mathrm{~N}^{\circ}$ & $\%$ & $\mathrm{~N}^{\circ}$ & $\%$ \\
\hline Bajo & 4 & 7 & 3 & 5 & 1 & 2 & 8 & 14 \\
\hline Medio & 2 & 3 & 9 & 15 & 3 & 5 & 14 & 23 \\
\hline Alto & 0 & 0 & 10 & 17 & 28 & 46 & 38 & 63 \\
\hline TOTAL & 6 & 10 & 22 & 37 & 32 & 53 & 60 & 100 \\
\hline
\end{tabular}

Hipótesis general

Existe relación significativa del estilo de dirección y la calidad de la gestión educativa en la Institución Educativa Domingo Mandamiento Sipán-Hualmay 2017.

Tabla 5

Correlación Rho de Spearman de la hipótesis general

\begin{tabular}{lcr}
\hline Descriptivos & Simbolo & \multicolumn{1}{l}{ Valor } \\
\hline Correlación & Rho & 0,41 \\
Significancia bilateral & p - valor & 0,001 \\
Significancia estadística & alfa & 0,05 \\
Nivel de confianza & 1 - Valor & 0,95 \\
Número de encuestados & $\mathrm{N}$ & 60
\end{tabular}

Como $p$-valor $=0.001$ es menor al valor de alfa, se llega a la conclusión que existe relación significativa entre el estilo de dirección y la calidad de la gestión educativa en la Institución Educativa Domingo Mandamiento Sipán - Hualmay 2017.

H1: Existe relación significativa del estilo autoritario con la calidad de la gestión educativa en la Institución Educativa Domingo Mandamiento Sipán - Hualmay 2017 
Tabla 6

Correlación Rho de Spearman de la hipótesis especifica 1

\begin{tabular}{lcr}
\hline Descriptivos & Simbolo & Valor \\
\hline Correlación & Rho & 0,46 \\
Significancia bilateral & p - valor & 0 \\
Significancia estadística & alfa & 0,05 \\
Nivel de confianza & 1 - Valor & 0,95 \\
Número de encuestados & $\mathrm{N}$ & 60 \\
& & \\
\hline
\end{tabular}

Como $p$-valor $=0.000$ es menor al valor de alfa, se llega a la conclusión que existe relación significativa entre el estilo autoritario con la calidad de la gestión educativa en la Institución Educativa Domingo Mandamiento Sipán - Hualmay 2017.

H2: Existe relación significativa del estilo democrático y la calidad de gestión educativa en la Institución Educativa Domingo Mandamiento Sipán - Hualmay 2017.

Tabla 7

Correlación Rho de Spearman de la hipótesis especifica 2

\begin{tabular}{lcr}
\hline Descriptivos & Simbolo & Valor \\
\hline Correlación & Rho & 0,32 \\
Significancia bilateral & p - valor & 0,002 \\
Significancia estadística & alfa & 0,05 \\
Nivel de confianza & 1 - Valor & 0,95 \\
Número de encuestados & $\mathrm{N}$ & 60
\end{tabular}

Como $p$-valor $=0,000$ es menor al valor de alfa, se llega a la conclusión que existe relación significativa entre el estilo democrático con la calidad de la gestión educativa en la Institución Educativa Domingo Mandamiento Sipán - Hualmay 2017.

H4: Existe relación significativa del estilo liberal y la calidad de la gestión educativa en la Institución Educativa Domingo Mandamiento Sipán-Hualmay 2017.

Tabla 8. Correlación Rho de Spearman de la hipótesis especifica 3

Como $p$-valor $=0,000$ es menor al valor de alfa, se llega a la conclusión que existe relación significativa entre el estilo liberal con la calidad de la gestión educativa en la Institución Educativa Domingo Mandamiento Sipán - Hualmay 2017.

\begin{tabular}{lcr}
\hline Descriptivos & Simbolo & \multicolumn{1}{c}{ Valor } \\
\hline Correlación & Rho & 0,60 \\
Significancia bilateral & $\mathrm{p}$ - valor & 0 \\
Significancia estadística & alfa & 0,05 \\
Nivel de confianza & 1 - Valor & 0,95 \\
Número de encuestados & $\mathrm{N}$ & 60 \\
& & \\
\hline
\end{tabular}

Como $\mathrm{p}$-valor $=0,000$ es menor al valor de alfa, se llega a la conclusión que existe relación significativa entre el estilo liberal con la calidad de la gestión educativa en la Institución Educativa Domingo Mandamiento Sipán - Hualmay 2017.

\section{DISCUSIÓN}

El resultado obtenido al contrastar la hipótesis general, mediante la prueba no paramétrica Rho de Spearman $(0,41)$ a un nivel de significancia del 0,05 nos permitió evidenciar estadísticamente que existe relación significativa del estilo de dirección y la calidad de la gestión educativa en la Institución Educativa Domingo Mandamiento Sipán-Hualmay-2017.

Se determinó que el $10 \%$ de los docentes consideraron una gestión educativa baja, el $37 \%$ medio y el $53 \%$ alto. Por otro lado, al analizar el estilo de dirección se determinó que el 13\% de los docentes lo percibieron como bajo, el $27 \%$ medio y el $60 \%$ alto. Estos resultados son concordantes con la investigación presentada por Arana (2017) quien demostró que existe una relación directa y significativa entre el liderazgo directivo y el desempeño docente. Por otra parte al evaluar la gestión de la calidad educativa. Sorados (2010) comprobó que el liderazgo de los directores se relaciona con la calidad de la gestión educativa de las instituciones educativas.

Al contrastar la hipótesis específica 1, se observó que el valor de la prueba no paramétrica Rho de Spearman $(0,46)$ a un nivel de significancia del 0,05 nos permitió evidenciar estadísticamente que existe relación significativa del estilo autocrático con la calidad de la gestión educativa en la Institución Educativa Domingo Mandamiento Sipán Hualmay-2017.

Al determinar el estilo de dirección autocrático el 10\% de los docentes percibieron como bajo, el $28 \%$ medio y el $62 \%$ como alto, es decir algunas veces el director toma decisiones sin hacer consultas a los docentes, algunas veces impone lo que se tiene que hacer y trata de forma respetuosa a los docentes. Debo mencionar que estos resultados son similares a los presentados por Raxuleu (2014) que demostró que existe una alta vinculación entre el liderazgo del director y el desempeño pedagógico del docente.

Al contrastar la hipótesis específica 2, se observó que el valor de la prueba no paramétrica Rho de Spearman $(0,38)$ a un nivel de significancia del 0,05 nos permitió evidenciar estadísticamente que existe relación significativa del estilo democrático y la calidad de la gestión educativa en la Institución Educativa Domingo Mandamiento Sipán-Hualmay-2017.

Al determinar el estilo de dirección democrático el $8 \%$ de los docentes lo percibieron como bajo, el $35 \%$ medio y el $57 \%$ como alto; es decir casi siempre el director toma en cuenta la participación e implicancia del docente en la toma de decisiones, llegando a generar una confianza entre ellos, resultado concordante con lo reportado por Gamarra (2011) quien comprobó que los estilos de gestión se relacionan con la calidad de la conducción de las instituciones educativas.

Por otro lado, el objetivo específico 3 consistió en determinar la relación del estilo liberal y la calidad de la gestión educativa en la Institución Educativa Domingo Mandamiento Sipán - Hualmay - 2017. Al contrastar la hipótesis específica 3 , se observó que el valor de la prueba no paramétrica Rho de Spearman $(0,60)$ a un nivel de significancia del 0,05 nos permitió 
evidenciar estadísticamente que existe relación significativa del estilo liberal y la calidad de la gestión educativa.

Al determinar el estilo de dirección liberal el 14\% de los docentes lo percibieron como bajo, el $23 \%$ medio y el $63 \%$ como alto; es decir siempre se ha observado en la institución la libertad de expresión y la delegación de responsabilidades que contribuyen a la oportuna toma de decisiones. Este resultado guarda relación con lo reportado por Ortiz (2014) quién manifiesta que el liderazgo pedagógico en los procesos de gestión educativa en los centros de educación básica es de vital importancia para planificar, llevar a cabo la transformación e innovación de los mismo para lograrla mejora de los aprendizajes en los alumnos.

\section{CONCLUSIONES}

Se comprobó estadísticamente que:

Existe relación significativa entre el estilo de dirección y la calidad de la gestión educativa en la Institución Educativa Domingo Mandamiento Sipán-Hualmay 2017.

Existe relación significativa entre el estilo autoritario con la calidad de la gestión educativa en la Institución Educativa Domingo Mandamiento Sipán - Hualmay 2017.

Existe relación significativa entre el estilo democrático con la calidad de la gestión educativa en la Institución Educativa Domingo Mandamiento Sipán - Hualmay 2017.

Existe relación significativa entre el estilo liberal con la calidad de la gestión educativa en la Institución Educativa Domingo Mandamiento Sipán-Hualmay 2017.

\section{REFERENCIABIBLIOGRÁFICA}

Arana, L. y Coronado, J. (2017). Liderazgo directivo y desempeño docente en una Institución Educativa Parroquial Distrito de San Isidro Lima - Perú. Tesis para Optar al Grado Académico de Maestro en Educación. Mención en Gestión Educativa. Escuela de Postgrado Universidad Marcelino Champagnat. Lima.

Ávila, R. (2010). Estadística Elemental. Lima, Perú: Estudio y Ediciones RA

Gamarra, I. A. (2011). Estilos de gestión de los directivos y su relación con la calidad de la conducción de las instituciones educativas de ex variante técnica de la UGEL $N^{\circ} 03$ de Lima. Tesis para optar el Grado Académico de Maestría en Gestión de la Educación. Facultad de Educación Unidad de Post-grado. Universidad Nacional Mayor de San marcos. Lima.

Gómez, V. G. (2013). Incidencia del estilo de gestión escolar en el clima institucional. Buenos Aires, Argentina. $\mathrm{O}$ b t e $\mathrm{n}$ i d o d e http://imgbiblio.vaneduc.edu.ar/fulltext/files/TC1 130 15.pdf
Ortiz, B.A. (2014). El liderazgo pedagógico en los procesos de gestión educativa en los centros de educación básica: Juan Ramón Molina, Las Américas Nemecia Portillo y José Cecilio del Valle, Municipio del Distrito Central. Tesis para obtener el Título de Máster en Gestión de la Educación. Universidad Pedagógica Nacional Francisco Marazán. Dirección de Posgrado. Tegucigalpa.

Tamayo y Tamayo, M. (2003). El proceso de la investigación científica. México D. F.: Limusa S.A.

Raxuleu, M. (2014). Liderazgo del director y desempeño pedagógico Docente. Tesis de Grado. Licenciatura en Pedagogía con orientación en Administración y Evaluación Educativas Facultad de Humanidades Universidad Rafael Landívar. Quetzaltenango. Guatemala.

Sorados, P. M. M. (2010). Influencia del liderazgo en la calidad de la gestión educativa. Tesis para optar el grado de Magíster en Educación con Mención en Gestión de la Educación. Facultad de Educación. Universidad nacional Mayor de San Marcos, Lima. 